\title{
Article
}

\section{Physics of Eclipsing Binaries: Heartbeat Stars and Tidally Induced Pulsations}

Pavlovski, K., Hambleton, Kelly, Degroote, P., Conroy, K., Bloemen, S., Kurtz, Donald Wayne, Thompson, S.E., Fuller, J., Giammarco, J., Pablo, H., Prša, A., Tkachenko, A. and Torres, G.

Available at http://clok.uclan.ac.uk/14587/

Pavlovski, K., Hambleton, Kelly, Degroote, P., Conroy, K., Bloemen, S., Kurtz, Donald Wayne ORCID: 0000-0002-1015-3268, Thompson, S.E., Fuller, J., Giammarco, J. et al (2013) Physics of Eclipsing Binaries: Heartbeat Stars and Tidally Induced Pulsations. EAS Publications Series, 64 . pp. 285-294. ISSN $1633-4760$

It is advisable to refer to the publisher's version if you intend to cite from the work. http://dx.doi.org/10.1051/eas/1364039

For more information about UCLan's research in this area go to http://www.uclan.ac.uk/researchgroups/ and search for < name of research Group>.

For information about Research generally at UCLan please go to http://www.uclan.ac.uk/research/

All outputs in CLoK are protected by Intellectual Property Rights law, including Copyright law. Copyright, IPR and Moral Rights for the works on this site are retained by the individual authors and/or other copyright owners. Terms and conditions for use of this material are defined in the policies page. 


\title{
PHYSICS OF ECLIPSING BINARIES: HEARTBEAT STARS AND TIDALLY INDUCED PULSATIONS
}

\author{
K. Hambleton ${ }^{1,2}$, P. Degroote ${ }^{3}$, K. Conroy ${ }^{4}$, S. Bloemen ${ }^{3}$, D. Kurtz ${ }^{2}$, \\ S.E. Thompson ${ }^{5}$, J. Fuller ${ }^{6}$, J. Giammarco ${ }^{7}$, H. Pablo ${ }^{8}$ and A. Prša ${ }^{1}$
}

\begin{abstract}
Heartbeat stars are a relatively new class of eccentric ellipsoidal variable first discovered by Kepler. An overview of the current field is given with details of some of the interesting objects identified in our current Kepler sample of 135 heartbeats stars. Three objects that have recently been or are undergoing detailed study are described along with suggestions for further avenues of research. We conclude by discussing why heartbeat stars are an interesting new tool to study tidally induced pulsations and orbital dynamics.
\end{abstract}

\section{Introduction}

Heartbeat stars are a relatively new class of eccentric ellipsoidal variables that show a unique periastron variation in the form of a tidal pulse. This variation arises from two processes: the gravitational interaction between the two components at periastron that causes the deformation of the stellar shape, and the mutual irradiation of the stars. The shape of the periastron variation is dependent on the inclination of the binary orbit, the eccentricity and the argument of periastron. Specifically, in the light curve, the inclination dictates the peak to trough ratio

\footnotetext{
1 Department of Astrophysics and Planetary Science, Villanova University, 800 Lancaster Ave., Villanova, PA 19085, USA

2 Jeremiah Horrocks Institute, University of Central Lancashire, Preston, PR1 2HE, UK

${ }^{3}$ Instituut voor Sterrenkunde, KU Leuven, Celestijnenlaan 200D, 3001 Leuven, Belgium

${ }^{4}$ Department of Physics and Astronomy, Vanderbilt University, VU Station B 1807, Nashville, TN 37235, USA

5 NASA Ames Research Center, Moffett Field, CA 94035, USA

6 California Institute of Technology, TAPIR 350-17, 1200 E. California Boulevard, Pasadena, CA 91125-0001, USA

7 Department of Astronomy and Physics, Eastern University, Saint Davids, PA 19087, USA

8 Département de Physique, Université de Montréal, CP 6128, Succursale Centre-Ville, Montreal, QC, H3C 3J7, Canada
} 


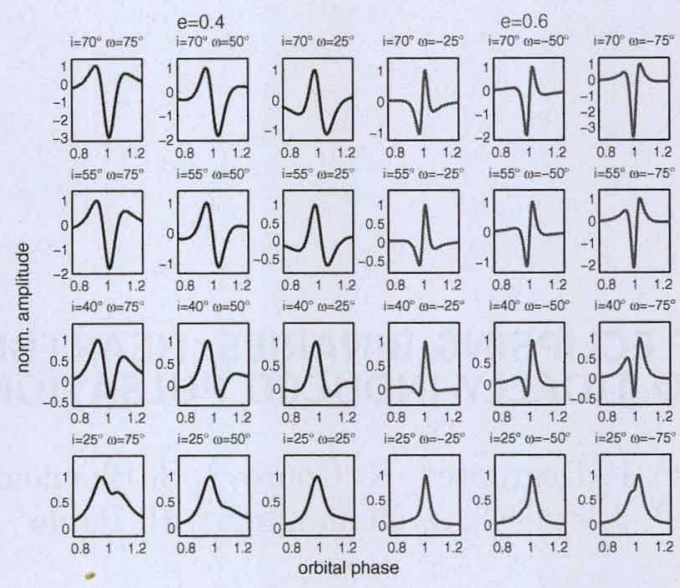

Fig. 1. Synthetic light curves generated from the analytic model of Kumar et al. (1995) for tidal distortions in an eccentric binary. The inclination (i) increases vertically and periastron angle $(\omega)$ increases horizontally. The relative flux of each curve has been normalized by the largest change in brightness. Since the grid is symmetric around $\omega=0$, the models are presented at two eccentricities, 0.6 and 0.4 (red and blue lines, respectively). Figure adopted from Tompson et al. (2012).

of the tidal pulse, the eccentricity determines its relative width and the argument of periastron determines the symmetry, as seen in Figure 1. The magnitude of the variation is determined by the separation of the objects, their masses and the gravity darkening exponent (see Sect. 2.3 for further discussion on the gravity brightening exponent).

The first observations of heartbeat stars were performed using the Kepler satellite, with the iconic heartbeat star, KOI-54 (Welsh et al. 2011; Fuller \& Lai 2011; Burkart et al. 2011), being the first of its kind. Shortly following, a catalogue of 17 heartbeat stars found in the Kepler data was published by Thompson et al. (2012) and 7 heartbeat stars detected using the OGLE ground based project by Nicholls \& Wood (2012). Following the efforts of the Heartbeat Star Team, the Kepler Eclipsing Binary Working Group, KASC, the Planet Hunters and several individuals, from the Kepler data alone we now have a list of 135 heartbeat stars, with the numbers still rising.

The Kepler sample of heartbeat stars is an extensive range of objects from main-sequence stars to red giants; naturally pulsating stars; objects with interesting orbital dynamics, i.e., rapid apsidal motion and increasing eclipses; stars that show frequency modulation (Shibahashi \& Kurtz 2012), objects with P-Cygni profiles in their spectral lines; and objects with tidally induced pulsations. At the precision of Kepler, we have found $\sim 20 \%$ of our sample pulsate with tidally induced pulsations. Interestingly, in our sample we found that the majority of main-sequence objects have spectral type $\mathrm{F}$, while all the low temperature objects are red giants. Figure 2 depicts a histogram of all the Kepler heartbeat stars with 


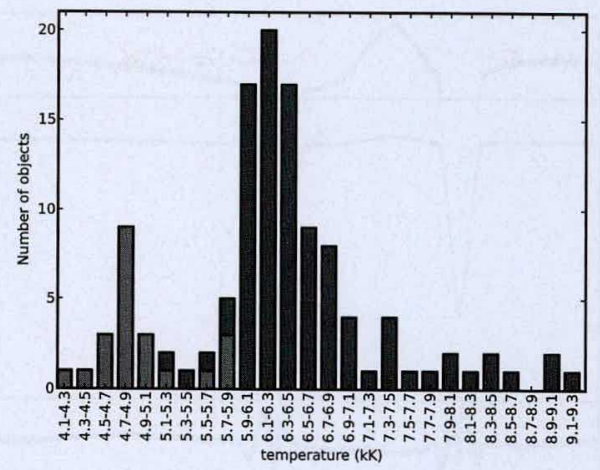

Fig. 2. A histogram of all the Kepler heartbeat stars as a function of temperature. The blue and the red bars represent the main sequence stars and red giants, respectively. The effective temperatures and $\log g$ values were taken from the Kepler Input Catalog. Only those with known temperatures are displayed.

known temperatures and $\log g$ values (taken from the Kepler Input Catalogue). The objects have been separated into main-sequence objects ( $\log g>3.7$ - blue) and red giants $(\log g \leq 3.7-$ red).

In 1995, Kumar, Ao \& Quataert (1995) introduced the theory of tidal interactions in eccentric ellipsoidal variables. They stated that two terms shape the light curve: the static term, which describes the stellar deformation and heating and the oscillatory term, which describes the tidally excited pulsations. Kumar et al. theorised that tidally induced pulsations will be driven if an eigenfrequency of one of the components in a binary system is close to the forcing frequency of the orbit (or its multiple). He also noted that an important aspect for the tidal excitation of modes is the periastron duration. He created a parameter. $b_{\alpha}$, which is the ratio of the mode frequency to the angular velocity of the stars at periastron (see Eq. (44), Hut 1981) when $b_{\alpha} \approx 1$, pulsation amplitudes are approximately equal to the static term, when $b_{\alpha}>$ a few, pulsation amplitudes are small. Finally, as high overtone $\mathrm{g}$-modes have little overlap with the tidal forcing function, intermediate overtone g modes (with $b_{\alpha} \approx 1$ ) are expected to have the highest amplitude tidally induced pulsations.

\section{Objects under detailed study}

\subsection{KIC 4544587}

KIC 4544587 (Hambleton et al. 2013) is an eccentric eclipsing binary system with self excited pressure (p) modes, tidally induced gravity (g) modes, g modes excited by three mode resonance and tidally influenced p modes ( $c f$. Fig. 3). It contains a late A type primary star and an early F type secondary star in a $2.189 \mathrm{~d}$ orbit with an eccentricity of 0.28 . This interesting object is also undergoing rapid apsidal motion with a cycle period of $182 \mathrm{y}$. 


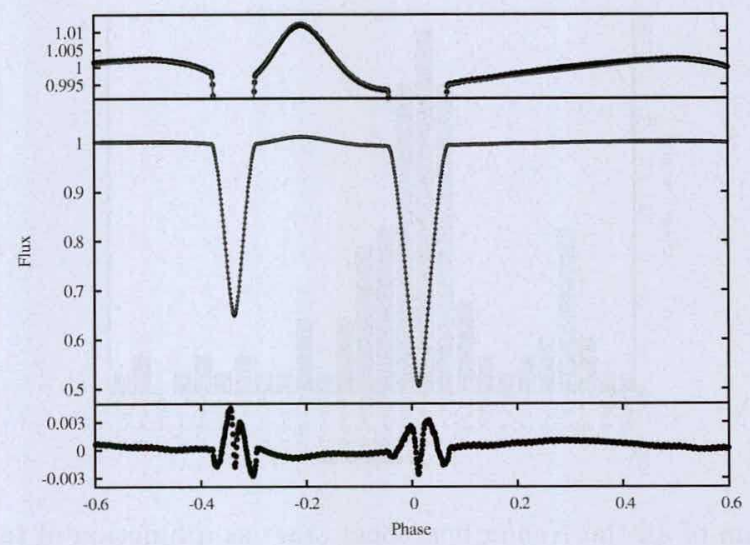

Fig. 3. Middle panel: theoretical PHOEBE model (red line) and observed light curve of KIC 4544587, prewhitened with pulsation frequencies with amplitudes $>0.04 \times 10^{-3}$ relative flux units (black points) for the short cadence data of Quarters 7 and 8. Lower panel: the residuals (black points) of the best-fit model (note the change of scale). Upper panel: a magnified image of the out of eclipse data and PHOEBE model fit.

A binary model of KIC 4544587 was created using PHOEBE (Prša \& Zwitter 2005) which is based on the Wilson-Devinney code (Wilson 1979). An iterative approach was performed where the binary model was subtracted from the original data to analyse the pulsations in the residuals. The pulsations were then prewhitened from the original data following which a new binary model was fitted to the prewhitened data. These steps were repeated until further repetitions no longer improved the binary model fit. A final binary solution was generated using the light curve and radial velocity data. This was combined with the disentangled spectra of the two components to determine their masses, $1.98 \pm 0.07 M_{\odot}$ and $1.60 \pm 0.06 M_{\odot}$, and radii, $1.76 \pm 0.03 R_{\odot}$ and $1.42 \pm 0.02 R_{\odot}$ alongside a detailed complement of fundamental and orbital parameters. The light curve model can be seen in Figure 3.

KIC 4544587 was first identified as an object with possible tidally induced pulsations, but was found to have a host of interesting features. As it contains stars in the $\delta$ Scuti and $\gamma$ Dor regions of the Hertzsprung-Russell diagram, we expected that the object would have $\mathrm{p}$ mode and $\mathrm{g}$ mode eigenfrequencies. When analysing the modes, however, it was found that 8 of the $14 \mathrm{~g}$ modes were multiples of the orbital frequency. These frequencies were ruled out as being the result of inadequate modelling, which can also result in frequencies at multiples of the orbital frequency, as the light curve clearly shows features that can only be described by pulsations. Furthermore, when the light curve is folded the pulsations remain, demonstrating that they are locked to the orbital period ( $c f$. Fig. 4).

The remaining g modes, which are not multiples of the orbital frequency, were identified as being part of a three mode resonance (Papaloizou \& Pringle 1981) indicative of non-linear tidal processes. Specifically, the addition of two modes 


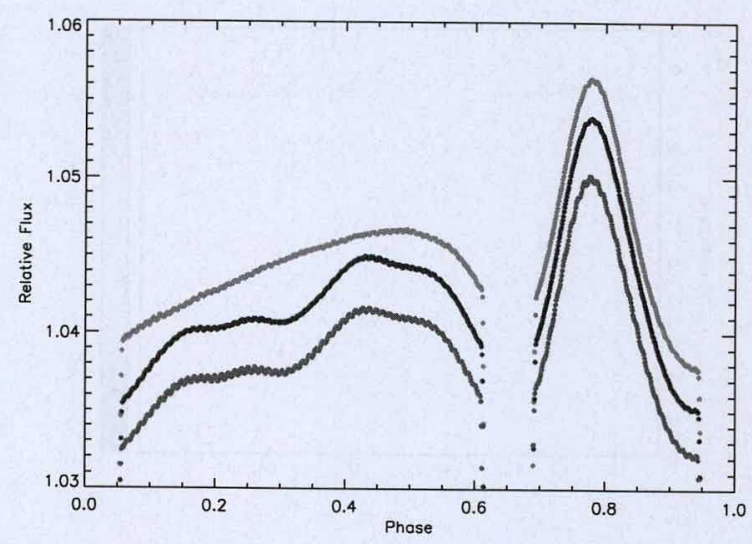

Fig. 4. A magnified image of the phase binned Kepler photometric short cadence light curve of KIC 4544587 for Quarters 7 and 8 with no frequencies prewhitened (pink, bottom curve), all the frequencies except those that are harmonics prewhitened (blue, middle curve) and all the frequencies prewhitened (green, top curve). The blue and pink light curves only demonstrate a minimal difference as all non-commensurate pulsations are cancelled out when the data is phase binned. The only explanation for the remaining variation in the blue and pink light curves is that they are resonantly excited pulsations. The light curves have been offset by 0.03 relative flux units for clarity.

equals a multiple of the orbital frequency (i.e. $f_{3}+f_{5}=12 \nu_{\text {orb }}$ ). KIC 4544587 presents two distinct types of non-linear driving for three mode resonance. One where the parent mode is the dynamical tide and is a clearly visible mode, and one where the parent mode is not visible, in which case the equilibrium tide is hypothesised to be the driving mechanism. The tidal nature of the $\mathrm{g}$ modes was predicted by the pulsational model preformed on KIC 4544587, which suggested that, given their masses, radii and temperatures, neither star should be unstable to $\mathrm{g}$ mode oscillations.

In the $\mathrm{p}$ mode region of $\mathrm{KIC} 4544587$, modes that were split by the orbital frequency but were not multiples of it, were found. Further investigation highlighted groupings of frequencies split by multiples of the orbital frequency. Figure 5 shows an echelle diagram, where the x-axis displays frequency modulo the orbital frequency. Here you can see clear vertical groups formed by frequencies that are split by the orbital frequency, but are not multiples of it. The current hypothesis for these frequencies are that the strongest peak in a group is a self excited p mode and that the remaining modes in that group are caused by non-linear interactions between the p mode and the tidally induced $\mathrm{g}$ modes.

\subsection{KIC 3749404}

KIC 3749404 is a double lined spectroscopic binary with a characteristic heartbeat star light curve and strong tidally induced pulsations ( $c f .6)$. Our preliminary 


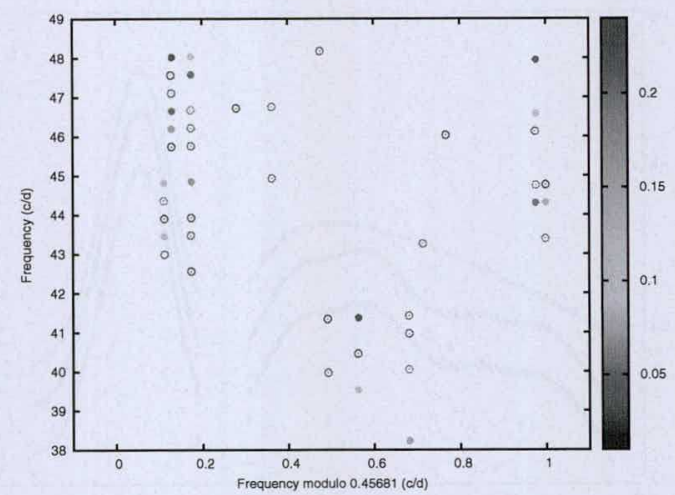

Fig. 5. An echelle diagram of the p-mode frequencies modulo the orbital frequency for KIC 4544587 using the short cadence Kepler data of Quarters 7 and 8. The points are coloured in terms of their amplitude in units of relative flux $\times 10^{-3}$ (see the key at the right of the figure). The filled circles are p-mode with amplitudes $>0.04$ parts per thousand (ppt) and the open circles are represent frequencies with amplitudes in the region $0.02-0.04 \mathrm{ppt}$, which are below the $3 \sigma$ confidence limit. The uncertainty in the frequencies is smaller than the points, thus not depicted. A high pass filter was applied to the $\mathrm{g}$-mode region prior to the identification of the p modes to remove the high frequency tails of the g-mode window pattern.

investigation of KIC 3749404 shows that the primary component is a slightly evolved star. As the secondary component is a mid-F star, this suggests that the primary component was an A or early $\mathrm{F}$ type star on the main sequence. The two objects are in a close orbit of $20.3 \mathrm{~d}$, with an eccentricity of $e=0.65$ and a periastron separation of $\sim 20 R_{\odot}$. KIC 3749404 has a rich frequency spectrum containing the static term (the deformation and reflection caused by the tides) and tidally induced pulsations. KIC 3749404 does not have any self-excited pulsations. This is an interesting feature of this object as the secondary component is in the $\gamma$ Dor instability strip, thus we expect it to pulsate through the mechanism of convective blocking. It has been previously noted that binarity can inhibit pulsations because stars rotate more slowly in binaries leading to less meridional mixing and thus diffusive settling of the heavier elements (Kurtz 1976), for example in the case of HD 181068 (Derekas et al. 2011). However, while this mechanism accounts for the reduction in modes that originate in ionisation zones, such as $\delta$ Sct p modes, it is not obvious why this mechanism would diminish modes driven by convective blocking that originates in the region where radiation turns to convection.

Due to the relatively large pulsations of KIC 3749404, the iterative separation of pulsations and binary information has proven to be difficult. This is because the pulsations and binary features are not separated in Fourier space as the pulsations begin at low multiples of the orbital frequency. This is further exacerbated by the similar amplitude of the binary and pulsation features. For this reason 


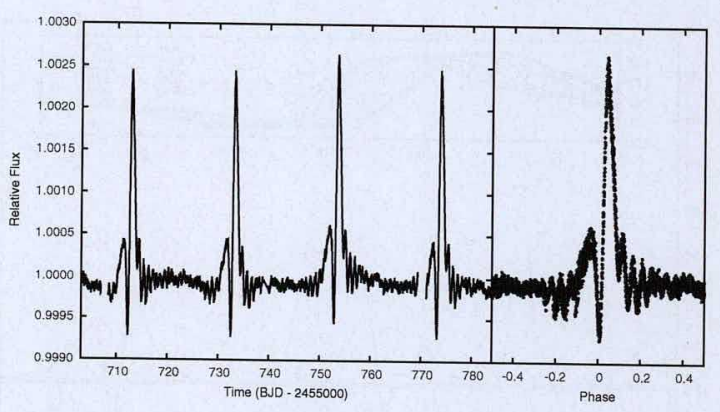

Fig. 6. The panels depict the Kepler long-cadence (Quarters 9-10) time series (left) and the phase folded light curve (right) of KIC 3749404. The dominant feature of the light curve, repeating once per orbital cycle $(20.3 \mathrm{~d})$, is the variability due to tidal deformation. Tidally excited modes are also clearly visible in the phase folded light curve due to their commencurability with the orbital period.

it is necessary to fit the pulsations and binary features simultaneously. This is currently possible by fitting sine curves and the binary model simultaneously, or for a more complete treatment of the stellar surface, by modelling the pulsations as perturbations to the Roche model, which can be preformed using the latest version of PHOEBE, PHOEBE 2.0.

The high eccentricity and tidally excited modes make KIC 3749404 an obvious candidate for the study of orbital evolution including apsidal motion. It has previously been suggested by Gies et al. (2012) that the rapid apsidal motion of KIC 4544587 could be attributed to its tidally induced pulsations. Willems \& Claret (2005) also suggested that apsidal motion could be increased by the presence of tidally induced pulsations. In the case of KIC 3749404 the apsidal advance can easily be seen over the duration of the $4 \mathrm{yr}$ life span of the Kepler mission. Figure 7 depicts the change in the light curve as the line of apsides rotates $360^{\circ}$, while the insert shows the motion over 4 years. By identifying the modes in KIC 3749404, it may be possible to provide observational evidence to substantiate these hypotheses.

\subsection{KIC 5006817}

KIC 5006817 is a heartbeat star with a red giant component in a $95 \mathrm{~d}$ orbit (cf. 8). The advantage of studying heartbeat stars with red giant components is that the radius and mass of the red giant can be determined using asteroseismology. Asteroseismology was performed on KIC 5006817 and gave the mass and radius of the primary component to be $1.49 \pm 0.06 M_{\odot}$ and $5.84 \pm 0.09 R_{\odot}$, respectively. A detailed model of the binary star characteristics was created using PHOEBE. As the secondary component has a mass of $0.29 \pm 0.03 M_{\odot}$, its luminosity is less than $1 \%$ of the total light of the system and thus the object is a single lined spectroscopic binary. For this reason the astroseismic values of the mass and radius were 


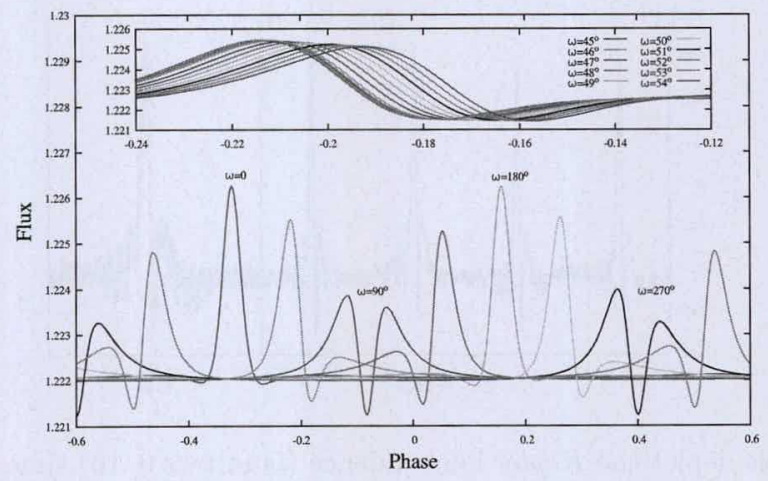

Fig. 7. The effect of the periastron value, $\omega$, on the shape of the periastron brightening of KIC 3749404 for one full cycle. The peaks change both their position in phase and their shape (width and amplitude) drastically with a varying values of $\omega$. The model demonstrated is of a Kepler Heartbeat star that exhibits one of the largest periastron advance rates ( $\sim 2^{\circ} / \mathrm{yr}$; Hambleton et al. 2014 , in preparation). Depicted in the inset is the effect on light curve shape over 4-yrs (the lifetime of the Kepler mission).

required to lift the large number of degeneracies in the light and radial velocity curve. This enabled the determination of the secondary star mass.

The magnitude of the periastron variation is a consequence of the masses and radii of the components, and the amount of gravity darkening the object experiences due to stellar rotation. Consequently, there is a degeneracy in the light curve between the mass ratio, the potentials (which scale as 1 /radius) and the gravity darkening exponents. The normally adopted value of the gravity darkening exponent for objects with convective outer envelopes is 0.32 (when applied to the temperature or 0.08 when applied to the flux). This is an empirically determined value for main sequence stars by Lucy (1967). When using this standard value, to obtain a good binary model fit the radius and mass of the primary star both deviated from their asteroseismic values by $3 \sigma$. While this may be considered plausible given the uncertainties on the temperature of the primary $(5000 \pm 250 \mathrm{~K})$ and the current debate about the level of uncertainties on the scaling relations, an important point to note is that the radius would have to increase by $3 \sigma$ and the mass decrease by $3 \sigma$ leading to an extreme discrepancy in the density, the most constrained asteroseismic parameter. As this is not an acceptable outcome, to resolve this issue, the gravity darkening exponent of the primary component (the only visible component in the light curve) was set to $1.0 \pm 0.03$. The most likely explanation is that the accepted gravity darkening exponent needs to be revised or completely mitigated from the models. Espinosa Lara \& Rieutord (2012) proposes a new treatment of gravity darkening that is a function of the Roche geometry of the star and is only lightly dependent on temperature, but heavily dependent on the stellar distortion (filling factor) and mass ratio. For KIC 5006817, Espinosa Lara \& Rieutord (2012) predict a gravity darkening value of $\sim 1.0$ for a detached system (with a fillout factor of $f=-2.61$ ) and a mass ratio of $0.199 \pm 0.001$. 


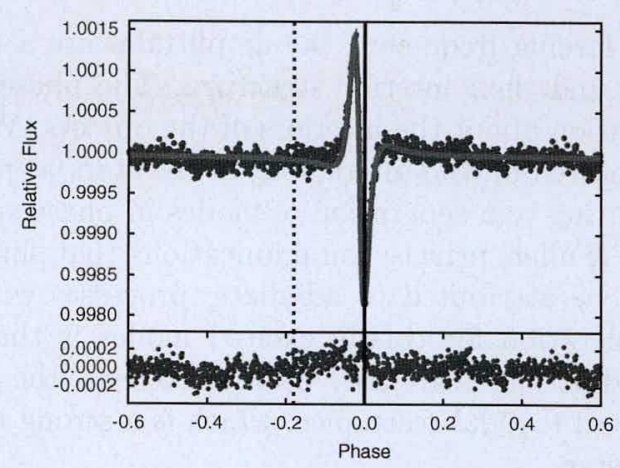

Fig. 8. Theoretical PHOEBE model of KIC 5006817 without beaming (red line) and observed light curve (black points) of the phased (94.82 d), binned Kepler long cadence data of Quarters 0-12. Lower panel: the residuals of the best-fit model. The dashed and solid lines are centred on the times of superior and inferior conjunction respectively.

Due to the configuration of KIC 5006817, with the objects having significantly different masses, the inclination angle being relatively high and the relatively high radial velocity of the objects at periastron, calculations show that Doppler Boosting should have a signature of $300 \mathrm{ppm}$ in the light curve. Doppler boosting is the combined effect of shifting the stars' spectral energy distributions with respect to the Kepler bandpass, aberration and an altered photon arrival rate.

When modelling the binary features, however, no signature of Doppler boosting was found. The possibility that the secondary component was more luminous than previously believed was ruled out by spectral disentangling, which placed an upper limit on the luminosity of the secondary to be $1 \%$ of the total luminosity. We also ruled out the possibility that the secondary is a continuum white dwarf, as the temperature would have to exceed $40000 \mathrm{~K}$, which would be visible in the light curve due to the reflection of the white dwarf off the red giant. Other than missing or incomplete physics in the binary models, the only conclusion postulated is that the Kepler satellite is not stable enough over long periods to preserve the beaming signal in the data. Further studies on beaming heartbeat stars will hopefully provide more conclusive evidence.

\section{Conclusion}

Heartbeat stars are an interesting class of binary star first discovered using the Kepler satellite. Although the modelling of the light curve can prove challenging due to the number of degeneracies and attributes such as tidally induced pulsations, these objects are broad in terms of the physics accessible through their study.

One of the most interesting attributes of heartbeat stars is the large number of them that pulsate with tidally induced pulsations. While the frequencies are 
dictated by the tidal forcing frequency, the amplitudes are a function of the periastron passage time and their internal structure. The phases of the pulsations may also hold information about the interiors of the objects. While it is theorised that the phases are centred on the interaction of the stars at periastron, observational evidence is pointing to a separation of modes in phase space. Furthermore, it has been suggested (Fuller, private communication) that slight deviations from expected phases may be attributed to adiabatic processes within the stellar interior. A final consideration for tidally excited modes is that some modes are significantly excited while others are not. As with KOI-54, the presence individual modes can be attributed to tidal resonance, which is a strong indicator about the eigenfrequencies of a star.

Another avenue of interest regarding heartbeat stars is orbital evolution. These stars are extreme test cases through which we can study apsidal motion, period changes through tides and how tidally induced pulsations affect the evolution of the orbit. The dynamical tide has been theorised to significantly affect the rate of apsidal advance. Heartbeat stars hold the key to confirming the current theories which suggest tidally induced pulsations causes the line of apsides to advance at an increased rate. Finally, the rate of apsidal advance is a consequence of the central density of the stellar components and as such holds information about the stellar structre and evolutionary state of a binary, which is obtainable through the study of heartbeat stars.

\section{References}

Burkart, J., Quataert, E., Arras, P., \& Weinberg, N.N., 2011 [arXiv]

Derekas, A., Kiss, L.L., Borkovits, T., et al., 2011, Science, 332, 216

Espinosa Lara, F., \& Rieutord, M., 2012, A\&A, 547, A32

Fuller, J., \& Lai, D., 2011, MNRAS, 420, 3126

Gies, D.R., Williams, S.J., Matson, R.A., et al., 2012, AJ, 143, 137

Hambleton, K., Kurtz, D.W., Prša, A., et al., 2013, MNRAS, 434, 925

Hut, P., 1981, A\&A, 99, 126

Kumar, P., Ao, C.O., \& Quataert, E.J., 1995, ApJ, 449, 294

Kurtz, D.W., 1976, ApJS, 32, 651

Lucy, L.B., 1967, Zeit. Astrof., 65, 89

Nicholls, C.P., \& Wood, P.R., 2012, MNRAS, 421, 2616

Papaloizou, J., \& Pringle, J.E., 1981, MNRAS, 196, 371

Prša, A., \& Zwitter, T., 2005, ApJ, 628, 426

Shibahashi, H., \& Kurtz, D.W., 2012, MNRAS, 422, 738

Thompson, S.E., Everett, M., Mullally, F., et al., 2012, ApJ, 753, 86

Welsh, W.F., Orosz, J.A., Aerts, C., et al., 2011, ApJS, 197, 4

Willems, B., \& Claret, A., 2005, ASP Conf. Ser., 333, 52

Wilson, R.E., 1979, ApJ, 234, 1054 
Copyright of EAS Publications Series is the property of Cambridge University Press and its content may not be copied or emailed to multiple sites or posted to a listserv without the copyright holder's express written permission. However, users may print, download, or email articles for individual use. 